Provided for non-commercial research and education use. Not for reproduction, distribution or commercial use.

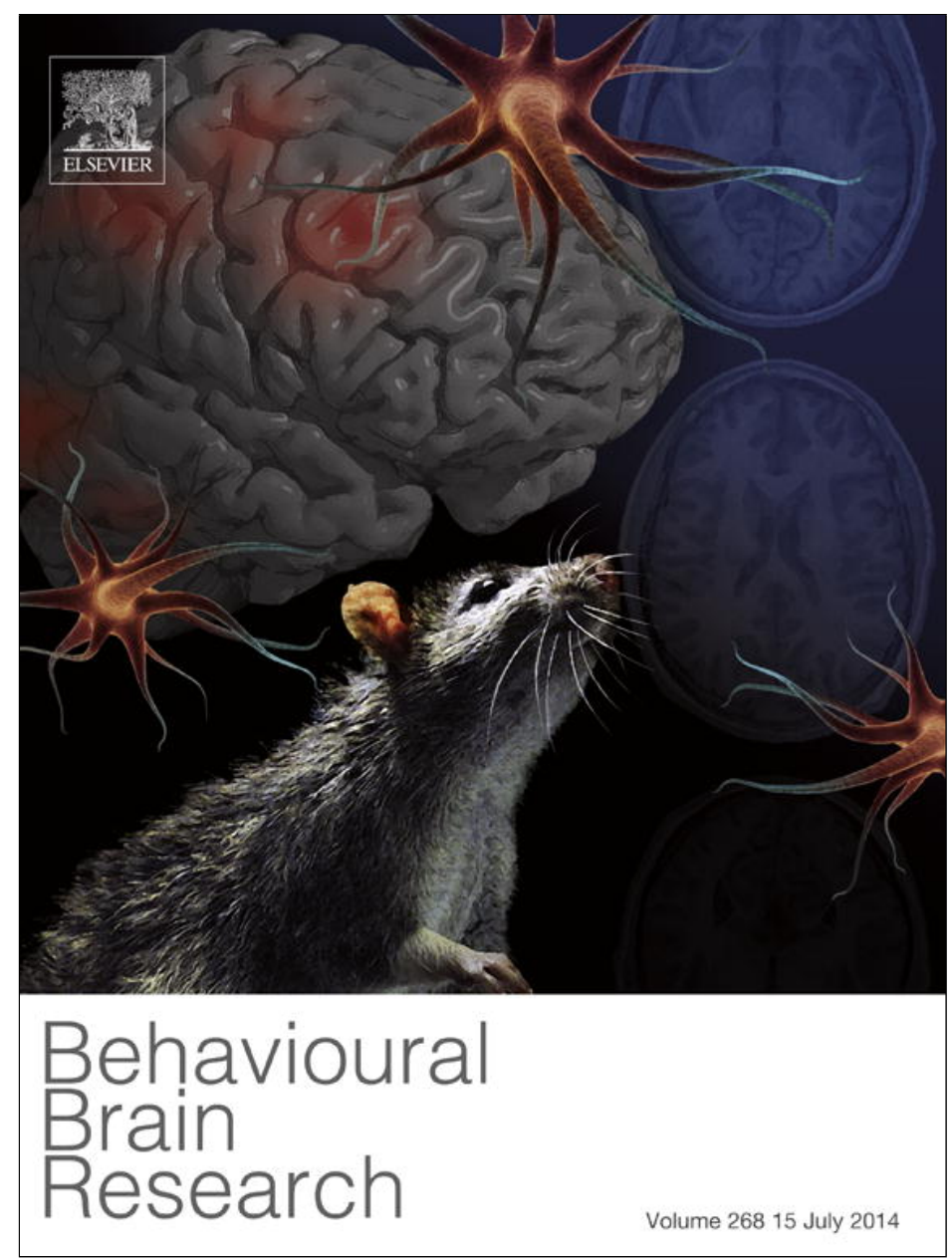

This article appeared in a journal published by Elsevier. The attached copy is furnished to the author for internal non-commercial research and education use, including for instruction at the authors institution and sharing with colleagues.

Other uses, including reproduction and distribution, or selling or licensing copies, or posting to personal, institutional or third party websites are prohibited.

In most cases authors are permitted to post their version of the article (e.g. in Word or Tex form) to their personal website or institutional repository. Authors requiring further information regarding Elsevier's archiving and manuscript policies are encouraged to visit:

http://www.elsevier.com/authorsrights 
Research report

\title{
Handedness as a marker of cerebral lateralization in children with and without autism
}

\author{
Gillian S. Forrester ${ }^{\mathrm{a}, *}$, Ruth Pegler ${ }^{\mathrm{a}}$, Michael S.C. Thomas ${ }^{\mathrm{b}}$, Denis Mareschal $^{\mathrm{b}}$ \\ a Department of Psychology, University of Westminster, 309 Regent Street, London, W1B 2HW England, United Kingdom \\ ${ }^{\mathrm{b}}$ Centre for Brain and Cognitive Development, Department of Psychological Sciences, Birkbeck University of London, Malet Street, London, WC1E 7HX \\ England, United Kingdom
}

\section{H I G H L I G H T S}

- We report on a detailed set of hand actions from two populations of right-handed boys.

- Typically developing boys demonstrated a right hand bias for actions to objects.

- Typically developing boys also demonstrated a left hand bias for actions to the self.

- Boys with autism revealed mixed-handedness for actions to objects and to the self.

- Handedness reveals a dissociation for functional specialization of the hemispheres.

\section{A R T I C L E I N F O}

\section{Article history:}

Received 31 October 2013

Received in revised form 19 March 2014

Accepted 24 March 2014

Available online 2 April 2014

\section{Keywords:}

Cerebral lateralization

Handedness

Autism

Self-directed behavior

\begin{abstract}
A B S T R A C T
We employed a multiple case studies approach to investigate lateralization of hand actions in typically and atypically developing children between 4 and 5 years of age. We report on a detailed set of over 1200 hand actions made by four typically developing boys and four boys with autism. Participants were assessed for unimanual hand actions to both objects and the self (self-directed behaviors). Individual and group analyses suggest that typically developing children have a right hand dominance for hand actions to objects and a left hand dominance for hand actions for self-directed behaviors, revealing a possible dissociation for functional specialization of the left and right hemispheres respectively. Children with autism demonstrated mixed-handedness for both target conditions, consistent with the hypothesis that there is reduced cerebral specialization in these children. The findings are consistent with the view that observed lateralized motor action can serve as an indirect behavioral marker for evidence of cerebral lateralization.
\end{abstract}

(C) 2014 Elsevier B.V. All rights reserved.

\section{Introduction}

There are different functional specializations of the left and right hemispheres for processing sensory information [see 1 for a review]. The division of labor between the two hemispheres is proposed to be an advantageous evolutionary adaptation found in both vertebrates [e.g. 2, 3] and invertebrates [e.g. 4] providing the brain with increased neural efficiency. Cerebral lateralization allows for disparate specialized processing to operate in parallel

\footnotetext{
* Corresponding author at: Department of Psychology, University of Westminster 309 Regent Street, London W1B 2HW, United Kingdom.

Tel.: +44 0207911 5000x69006; mobile: +44 07814704884 .

E-mail addresses: g.forrester@westminster.ac.uk, gilliansforrester@gmail.com (G.S. Forrester).
}

within the left and right hemispheres, which decreases the duplication of functioning across hemispheres and eliminates the initiation of simultaneous and incompatible responses [5-7]. Not only do the left and right hemispheres appear to have distinctive roles, the organization of the human brain is such that the innervations of the musculature that originate from the motor cortices extend contralaterally [8]. As a result, the left hemisphere controls the right side of the body and the right hemisphere controls the left side of the body. Thus, hemispheric specialization can manifest as contralateral physical actions [e.g. 9].

The most prominent examples of a shared lateral bias for human anatomical and functional hemispheric specialization is handedness, and the neural regions associated with speech production [e.g. inferior frontal gyrus: 10] and comprehension [superior temporal gyrus: 11]. It is commonly reported that the human population exhibits approximately $90 \%$ right-handedness [e.g. 12] and, within 
the right-handed population, approximately 95\% of individuals have language-processing regions situated in the left hemisphere of the brain [13]. Therefore, left hemisphere specialization is prominent in right-handed individuals [e.g. 14]. However, the existence of a left hemisphere dominance for both language processing and manual activities cannot presume that these cerebral asymmetries are correlated. Some studies have shown weak correlations between the strength of handedness and cerebral specialization for language in adults [15], and even an absence of a significant correlation between handedness for manipulative actions and language performance in very young children [e.g. 16, 17]. Additionally, $70 \%$ of left-handers also demonstrate left cerebral hemisphere dominance for language $[14,18]$, indicating a complex relationship between anatomical and functional hemispheric specialism. However, as there is no unified system for measuring handedness, it is also possible that differences in handedness patterns across studies may be symptomatic of the vast range of measurement techniques [e.g. 19].

Handedness is often assessed through subjective self-reporting and surveys [e.g. Edinburgh Handedness Inventory; 20], and has been defined using a variety of terms and measures across fields of study. Handedness is commonly considered to be the hand that is preferred for a specific task, regardless of performance, however it can also reflect hand efficiency with respect to speed and accuracy [e.g. 21]. Handedness can be categorized as right, left or mixed along a gradient that ranges from strongly left-handed to strongly right-handed [e.g. 19, 22]. It is generally established by the time typically developing children start school [23-25]. As in adult populations, associations have been drawn between hemispheric asymmetries associated with language and hand biases in children [26].

Some investigations of child handedness suggest that lefthandedness can be an indicator of decreased cerebral lateralization [e.g. 27-30]. However, other studies involving children, suggests that stronger hand dominance (left or right) correlates with both earlier language acquisition [31] and the successful hemispheric specialization for language [1]. For example, hand dominance (left or right) for manipulative tasks (e.g. drawing) has been associated with typical neurodevelopment, whereas inconsistent hand dominance has been associated with significantly lower developmental assessment scores in children, using the Viennese Development Test (WET) [32]. A growing body of evidence now indicates that reduced cortical lateralization is associated with impaired cognitive function and can manifest behaviorally as mixed-handedness [e.g. 33-37].

The frequency of mixed-handedness appears to rise within populations of individuals with autism (autistic spectrum disorder, ASD). ASD is a Pervasive Developmental Disorder [38], marked by symptoms that commonly include reduced language and social skills. Most children with ASD present impairments in receptive and expressive language [39], which can be the most obvious behavioral symptom of the disorder leading to a diagnosis [40]. Diagnosis is generally established in early childhood, but can be severely delayed when symptoms are subtle presenting alongside relatively intact language [e.g. 41]. While mixed-handedness makes up approximately $3-4 \%$ of the general population [e.g. 42], populations with ASD reveal mixed-handedness at proportions of between $17 \%$ and $47 \%$ [for a review see 43 ]. However, it has been reported that children with ASD who possess either left or right hand dominance, generally tend to have stronger language capabilities, compared with mixed-handed children with ASD. A further investigation suggests that in addition to language difficulties, mixed-handed children have a greater likelihood of having scholastic and mental health problems that persist into adolescence [44]. While a causal relationship between hand dominance and cognitive performance remains uncertain, measures of mixed-handedness could facilitate the recognition of children who are at risk for reduced cognitive function.

Recent evidence suggests that ASD is likely to have an early developmental onset characterized by hypo-lateralization of brain function for expressive and receptive language processes [45] long before there is visible behavioral evidence of language impairment [46]. Motor behaviors provide one possible area of exploration for further investigation. Motor capabilities have become a topical issue in the study of overt behavioral symptoms of children with ASD. It is now suspected that aberrant pruning during the development of ASD disrupts early sensory and motor processes [47], causing anomalies within these domains to become visible first. For example, infants with a familial risk for developing ASD have demonstrated significantly lower motor scores as early as 7 months of age [48]. A firm understanding of handedness strength across development for functionally specific tasks may afford a new approach to indirectly assess hypo-lateralization of brain function in children at risk for ASD.

Hand dominance has traditionally focused on school-aged children and left hemisphere dominant functions (e.g. object manipulation, right-handedness). In general, these studies have identified putative associations between hand dominance and cognitive performance on the basis of subjective parent-report, self-report or surveys for handedness. However, observational studies of naturalistic hand actions have demonstrated that hand dominance can be objectively revealed much earlier than preschool age [e.g. 24]. For example, right-handed dominance for manual tasks has been observed in typically developing infants between 6 months and 18 months of age [49-51]. Studies of observed naturalistic hand dominance in children, have observed actions such as pointing gestures, unimanual grasping of objects and bimanual tasks. However, hand dominance for different functional behaviors (e.g. communicative and non-communicative) have not been previously shown to be correlated in young children [e.g. 17, 49, 52-54]. In fact, a disparate range of experimental paradigms for assessing handedness in children has resulted in a variety of patterns of asymmetries depending hand action function [e.g. 16]. These studies showcase an opportunity for broader investigations of handedness across ages, revealing more complex patterns of handedness across development than previously found employing traditional reporting approaches. However, these studies also highlight the possibility that differences in handedness patterns across studies may be in part due to the vast range of paradigms and measurement techniques employed [e.g. 19].

In addition to early handedness evaluation, observing naturalistic handedness behaviors allows for the exploration of a more comprehensive range of hand behaviors. For example, the study of three preliterate cultures, using methods developed in ethology, revealed that the only condition under which spontaneous hand actions were preferentially lateralized across a pooled dataset of naturalistic hand actions was for object manipulation during tool use. Handedness for non tool-use actions, pooling a range of hand actions to both social partners (e.g. embrace) and to the self (e.g. nose wipe), demonstrated a propensity toward mixedhandedness [55]. The authors noted that traditional studies of handedness were narrowly defined and did not represent the naturalistic actions of daily life. A recent study of children also found that hand dominance varied across targets, even in those who are otherwise considered right-hand dominant by parent report [56]. The authors demonstrated that while typically developing right-handed boys (aged 4-5 years) expressed a significant right hand dominance for object manipulation, no hand preference was found for hand actions directed toward social partners and the self. The authors proposed that in typically developing children, hand actions to object and hand actions to the self/social partners are functionally different behaviors and as thus are associated 
with different patterns of hemispheric specialization. Specifically, the authors posited that while object manipulation revealed the expected left hemisphere/right hand dominance, hand actions directed to social partners and the self (pooled) incorporated additional right hemisphere resources for processing social-emotional content. This interpretation is consistent with prevailing theories of social-emotional processing in humans. In humans, the right hemisphere hypothesis considers the right hemisphere to be dominant in all forms of emotional expression and perception [e.g. 57], while the valence theory posits that the left hemisphere dominance is dominant for positive affect and right hemisphere dominance for negative affect [e.g. 58, 59].

Self-directed behaviors (SDBs) have been labeled by a host of names (e.g. self-adaptors [60], body manipulators [61]) and have a long history within the field of psychiatry. Evidence suggests a link between stress and SDBs. Specifically, the frequency of SDBs have been correlated with ratings of anxiety and guilt [62]. SDBs are considered to be adaptive responses to counteract stressors and facilitate a return to homeostasis [63]. To date, the influence of cerebral dominance associated with hand dominance for SDBs in humans has not been investigated. Compared with object manipulation, SDBs may represent a functionally different type of manual behavior and would benefit from further investigation separate from hand actions to social partners.

Observed naturalistic assessment of hand dominance presents certain challenges akin to that of the dense data approaches required for acquiring detailed observational information from individual cases of early language development [64]. Additionally, the fine-grained coding of corpus data sets is a time-consuming process that typically relies on small samples and case studies. Nevertheless, this approach is a data-rich process, necessary to advance our understanding of the association between neurodevelopment, behavior and prognosis. To date, disparities in findings from handedness studies highlight the fact that there is no existing systematic approach for the assessment for handedness. Additionally, there is a paucity of studies that observe naturalistic hand actions for different functional target-types across child populations, hindering our understanding of any underlying relationships between cerebral lateralization and hand preference.

The current study employed a behavioral observation technique (MultiDimensional Method, MDM) to investigate if handedness is influenced by target type, in typically and atypically developing boys. The MDM is a standardized, objective, coding framework to assess physical action within space, time and context [65]. The study was designed to systematically assess and compare the handedness actions of typically developing boys and boys with autism. We examined how the target type of a manual action influenced the hand with which a child chooses to interact with that target for both groups. Based on a previous naturalistic study child handedness [e.g. 56], we hypothesized that right-handed typically developing children hand choice of would vary depending on the targets type. Additionally, we hypothesized that children with autism would demonstrate a weaker pattern of hand dominance consistent with neuroscientific evidence of decreased lateral specialization in these individuals [45].

\section{Methods}

\subsection{Participants}

Four typically developing (TD) boys (mean age 57.8, SD 5.25 months; range 53-65 months) and four boys diagnosed with autism (mean age 60.8, SD 3.86 months; range 57-65 months) participated in the study. Chronologically age-matched children participated as part of an opportunity sampling of children who were all attending the same school. The test environment was unique in that the TD boys attended the mainstream portion of a primary school, and the boys diagnosed with autism attended the adjoining special needs section of the same school, dedicated to children with a clinical diagnosis of ASD. Based on a subjective report, parents were asked by letter to subjectively classify the children as left, right or mixedhanded. Additionally, teachers were verbally asked to corroborate parent classification of child handedness. All children chosen to participate in the study were classified as right-handed. Children of this age range were chosen because evidence suggests stable handedness [e.g. 24] and the cerebral processes associated with hand preference for unimanual actions have been established by three years of age $[49,66]$. However, strength of hand bias may continue to increase until approximately seven years of age [e.g. 67]. It is not unusual that our sample consisted of all boys, as there is strong evidence to suggest that more boys than girls are diagnosed with ASD. The ratio of male to females diagnosed with ASDs is at least 4:1, if not higher [e.g. 68]. All participants with ASD had an existing diagnosis of autism; a prerequisite for admittance to the special needs school. Original diagnoses were made through a variety of clinical assessments.

\subsection{Data capture}

The Multidimensional Method (MDM) was employed for data capture, coding and analyses in order to reveal structure from signals elicited through organism-environment interactions [65] and to facilitate direct comparisons with previous investigations [e.g. 56]. The MDM is based on the idea that physical actions are embedded within space, time and situational variables. It considers behavior as multimodal and builds on concepts of distributed cognition [69-71] to provide a bottom-up, noninvasive approach to the investigation of behavior. The current investigation employed a focal video sampling approach [72] in which one camera recorded a close-up view of a focal individual in order to capture fine-grained manual actions. Digital video cameras (Panasonic NVGS11B: UK; Sony DCR-TRV900E, IT) were tripod mounted, but mobile, and followed a child's activity using zoom, tilt and swivel to optimize their view. Video footage was collected at 24 frames per second. Video streams were compressed to 15 frames/s for subsequent coding.

All participants were filmed during the administration of the standardized Autism Diagnostic Observational Schedule (ADOS), conducted by a licensed clinician. The ADOS is a standardized diagnostic assessment that addresses the developmental level and age of the child through activities designed to elicit social interactions, communication and repetitive behaviors for the purpose of diagnosing ASD [73]. Although the ADOS was not designed to assess typical development, TD children were given the ADOS to standardize the situational environment within which the observational data were collected. Experimental data collection involved one continuous focal sampling session of each child during the participation of the clinical assessment. The time taken to assess each child varied depending on the performance of the child. All children completed the diagnostic assessment module whilst seated at a table in a quiet room and in the presence of only the clinician and the cameraperson. Observation times were between 20 and 35 min (Mean $=25.6, \mathrm{SD}=4.2$ ).

Each participant was administered the module of the ADOS that was considered appropriate for their level of verbal communication abilities. Modules ranged from 1 to 3 (1 for little or no phase speech, 2 for some phase speech but verbally non-fluent, 3 for verbally fluent). Three of four boys with autism were administered Module 1, while one boy with autism completed Module 2 . Three of four TD boys completed module 2, while one TD boy completed Module 3. All participants completed all elements of the ADOS test 
procedure. ADOS results confirmed a diagnosis of autism for all four boys placed within the ASD participation group.

\subsection{Behavioral coding}

Handedness assessments generally consist of either or both unimanual manipulations and bimanual activities. In bimanual activities, both hands are employed in a coordinated manner and are sensitive to task complexity. Bimanual activities have been reported to elicit stronger patterns of hand biases in children, adults [49,74-76] and non-human primates [for a review see 77]. However, unimanual actions have been shown to be sufficient to elicit patterns of hand bias for functionally different targets types in both non-human primates [78,79] and in children [56] and were most suitable in order to accommodate both of the target types for the present study. Unimanual actions were classified as single-handed lateralized (left, right) actions that acted upon, and made physical contact with the self or and inanimate object targets, while the other hand remained at rest. Any action where one hand was already engaged or was performing an act of posture support was excluded from the dataset.

Two types of unimanual hand actions were considered. First, contact with the self (self-directed behaviors; SDBs) consisted exclusively of manual actions directed toward and making contact with the individual's own body (e.g. supporting the head, scratching actions, nose wipes, eye rubs, hair and face and body manipulations). Manual actions directed toward other individuals in the room (e.g. the camera person, the clinician) were excluded. Actions to social partners combined with actions to the self have previously resulted in mixed-handedness in gorillas [78], chimpanzees [79] and children [56]. As the present testing environment did not afford social partners, the investigation provided a unique opportunity to isolate and investigate SDBs as separate from actions to social partners. Second, inanimate object targets comprised of all forms of manual contact with objects in the room (e.g. touching, grasping, pushing). Object targets were classified as either loose or fixed non-living items. However, manual contact with the table at which participants were seated was excluded from analysis due to the high probability of manual contact being made with this target whilst interacting with objects situated on the table. Additionally, communicative hand actions, where a gesture was performed but no physical contact was made with the self or an object, were also excluded from data capture (e.g. pointing). The task performed by the hand was not considered, only the nature of the target itself.

There is some discussion within the primate literature about whether bouts or frequencies (events) of hand actions constitute the most accurate measure of manual bias for statistical analysis $[80,81]$. Frequencies have raised concerns for experiments investigating bimanual actions, (e.g. bimanual feeding) because these actions tend to develop into sequences, thus violating an independent choice of two hands for actions subsequent to the initial dominant hand choice [e.g. pseudo-replication: see 82, 83]. Because both hands were required to be 'at rest' for the coding of unimanual actions, we preserved independence of the two hands. Thus, the measures reported here represent the more conservative measure of bouts. A unimanual hand frequency count was attributed to an action in which the child reached and made contact with the target.

\subsection{Data analyses}

Analyses focused on the handedness of individual children using a dense data set of naturalistic manual actions. Though participant numbers were small due to the dense data approach, group comparisons were also considered.

\subsubsection{Case analyses}

Handedness index (HI) scores and binomial approximations to the $z$-scores were calculated to highlight individual participant patterns. Handedness Index $(\mathrm{HI})$ scores were calculated using the formula $[H I=(R-L) /(R+L)]$, with $R$ and $L$ being the frequency counts for right and left hand dominance for unimanual actions respectively. HI values vary on a continuum between -1.0 and +1.0 , where the sign indicates the direction of hand preference. When $\mathrm{R}=\mathrm{L}$, then HI is zero. Positive values reflect a right hand preference while negative values reflect a left hand preference. The absolute value depicts the strength of hand preference. The directional strength of hand preference for each participant was calculated using $z$-scores such that children were left handed when $z \leq-1.96$, right handed when $z \geq 1.96$ and ambiguously handed when $-1.96<z<1.96$. Binomial tests were performed for each individual, in order to indicate whether the use of the left and right hands significantly differed for SDBs and actions toward inanimate objects. Alpha was set at 0.05 and all tests were two-tailed.

\subsubsection{Group analyses}

Group analyses were conducted using Fisher's exact tests. Paired-sample $t$ tests were used to test simple effects. Because all participants were observed during the same diagnostic assessment, statistical calculations were performed on raw frequencies of manual actions. However, proportions were also calculated for each participant to equalize the weighting that each participant contributed to the data set. Proportions were calculated by dividing the frequency of left or right hand actions by the total frequency of actions.

\section{Results}

\subsection{Case analyses}

Based on parent and teacher reports, all 8 participants were right-handed. Raw frequencies, binomial approximations of $z$ scores for each participant $(P)$ by lateralized target condition (object, SDBs), HI scores and hand classification are presented in Table 1.

\subsubsection{Unimanual actions to inanimate targets}

3.1.1.1. Typically developing children: case analyses. TD1 produced 28 left-handed and 105 right-handed actions toward inanimate objects resulting in a right hand HI score of 0.58 and a significant right hand bias $(p<.001)$, based upon the binomial approximation of the $z$-score $(z=6.59)$. Results from analyses of TD2, TD3 and TD4 handedness followed the same pattern. TD2 produced 35 left-handed and 83 right-handed actions toward inanimate objects resulting in a right hand $\mathrm{HI}$ score of 0.41 and a significant right hand bias $(p<.001)$, based upon the binomial approximation of the $z$ score $(z=4.33)$. TD3 produced 55 left-handed and 101 right-handed actions toward inanimate objects resulting in a right hand HI score of 0.29 and a significant right hand bias $(p<.001)$, based upon the binomial approximation of the $z$-score $(z=3.61)$. TD4 produced 43 left-handed and 86 right-handed actions toward inanimate objects resulting in a right hand $\mathrm{HI}$ score of 0.33 and a significant right hand bias $(p<.001)$, based upon the binomial approximation of the $z$-score $(z=3.70)$.

3.1.1.2. Children with autism spectrum disorder: case analyses. AS1 produced 51 left-handed and 68 right-handed actions toward inanimate targets resulting in a mixed-handedness HI score of 0.14 and no significant hand preference $(p<.142)$, based upon the binomial approximation of the $z$-score $(z=1.47)$. AS2 produced 49 left-handed and 53 right-handed actions toward inanimate objects resulting in a mixed-handedness $\mathrm{HI}$ score of 0.04 and no hand 
Table 1

Frequencies, binomial approximations of the $z$-score and HI scores of unimanual lateralized hand actions to the self and to objects.

\begin{tabular}{|c|c|c|c|c|c|c|c|c|c|c|c|c|}
\hline \multirow[t]{2}{*}{ Participant } & \multicolumn{6}{|c|}{ Unimanal actions to objects } & \multicolumn{6}{|c|}{ Unimanual actions to self } \\
\hline & Left $F(P)$ & Right $F(P)$ & $\begin{array}{l}\text { Binomial } \\
\text { score }\end{array}$ & $z$-Score & $\begin{array}{l}\text { HI } \\
\text { score }\end{array}$ & $\begin{array}{l}\text { Hand } \\
\text { Class. }\end{array}$ & Left $F(P)$ & Right $F(P)$ & $\begin{array}{l}\text { Binomial } \\
\text { Score }\end{array}$ & $z$-Score & $\begin{array}{l}\text { HI } \\
\text { Score }\end{array}$ & $\begin{array}{l}\text { Hand } \\
\text { Class. }\end{array}$ \\
\hline TD 1 & $28(.15)$ & $105(.57)$ & $<0.001^{*}$ & 6.59 & 0.58 & Right & $40(.22)$ & $12(.06)$ & $<0.001^{*}$ & -3.74 & -0.54 & Left \\
\hline TD 2 & $35(.23)$ & $83(.54)$ & $<0.001^{*}$ & 4.33 & 0.41 & Right & $22(.14)$ & 14(.09) & 0.242 & -1.17 & -0.22 & Mixed \\
\hline TD 3 & $55(.22)$ & $101(.40)$ & $<0.001^{*}$ & 3.61 & 0.30 & Right & $68(.27)$ & $28(.11)$ & $<0.001^{*}$ & -3.98 & -0.42 & Left \\
\hline TD 4 & $43(.26)$ & $86(.52)$ & $<0.001^{*}$ & 3.70 & 0.33 & Right & $31(.19)$ & $5(.03)$ & $<0.001^{*}$ & -4.17 & -0.72 & Left \\
\hline ASD 1 & $51(.31)$ & $68(.41)$ & 0.142 & 1.47 & 0.14 & Mixed & $21(.13)$ & $24(.15)$ & 0.764 & 0.30 & 0.07 & Mixed \\
\hline ASD 2 & $49(.39)$ & $53(.42)$ & 0.764 & 0.30 & 0.04 & Mixed & $10(.08)$ & $13(.10)$ & 0.674 & 0.42 & 0.13 & Mixed \\
\hline ASD 3 & $69(.53)$ & $42(.32)$ & $0.014^{*}$ & -2.47 & -0.24 & Left & $10(.08)$ & $9(.07)$ & 1.000 & 1.00 & -0.05 & Mixed \\
\hline ASD 4 & 11(.19) & $26(.44)$ & $0.021^{*}$ & 2.30 & 0.41 & Right & $12(.20)$ & $10(.17)$ & 0.834 & -0.21 & -0.09 & Mixed \\
\hline
\end{tabular}

$p<0.05$, TD: typically developing, ASD: autism spectrum disorder, $F$ : frequency, $P$ : proportion, HI score: handedness index, Hand Class.: hand classification based on binomial significance.

preference significant $(p<.764)$, based upon the binomial approximation of the $z$-score $(z=0.30)$. AS3 produced 69 left-handed and 42 right-handed actions toward inanimate objects resulting a lefthanded HI score of -0.24 and a significant left hand bias $(p<0.014)$, based upon the binomial approximation of the $z$-score $(z=-2.47)$. AS4 produced 11 left-handed and 26 right-handed actions toward inanimate objects resulting in a right hand $\mathrm{HI}$ score of 0.41 and a significant right hand bias $(p<0.021)$, based upon the binomial approximation of the $z$-score $(z=2.30)$.

\subsubsection{Unimanual self-directed behaviors}

3.1.2.1. Typically developing children: case analyses. TD1 produced 40 left-handed and 6 right-handed SDBs resulting in a left-handed HI score of -0.54 and a significant left hand bias $(p<.001)$, based upon the binomial approximation of the $z$-score $(z=-3.74)$. TD3 and TD4 followed the same pattern. TD3 produced 68 left-handed and 28 right-handed SDBs resulting in a left-handed HI score of -0.42 and a significant left hand bias $(p<.001)$, based upon the binomial approximation of the $z$-score $(z=-3.98)$. TD4 produced 31 left-handed and 5 right-handed SDBs resulting in a left-handed HI score of -0.72 and a significant left hand bias $(p<.001)$, based upon the binomial approximation of the $z$-score $(z=-4.17)$. TD2 produced 22 left-handed and 14 right-handed SDBs resulting in a mixed-handedness $\mathrm{HI}$ score of -0.22 and a non-significant left hand preference $(p=0.242)$ based upon the binomial approximation of the $z$-score $(z=-1.17)$.

3.1.2.2. Children with autism spectrum disorder: case analyses. AS1 produced 21 left-handed and 24 right-handed SBDs resulting in a mixed-handedness HI score of 0.07 and no significant hand preference $(p=.764)$, based upon the binomial approximation of the $z$-score $(z=0.30)$. AS2 produced 10 left-handed and 13 right-handed SBDs resulting in a mixed-handedness $\mathrm{HI}$ score of 0.13 and no significant hand preference $(p=.674)$, based upon the binomial approximation of the $z$-score $(z=0.42)$. AS3 produced 10 lefthanded and 9 right-handed SBDs resulting a mixed-handedness HI score of -0.05 and no significant hand preference $(p=1.000)$, based upon the binomial approximation of the $z$-score $(z=1.00)$. AS4 produced 12 left-handed and 10 right-handed SBDs resulting in a mixed-handedness $\mathrm{HI}$ score of -0.09 and no significant hand preference $(p=.834)$, based upon the binomial approximation of the $z$-score $(z=-0.21)$.

\subsection{Group analyses}

\subsubsection{Typically developing children}

Mean Handedness Index scores (MHI) were calculated for target categories and overall strength of handedness (Fig. 1). Typically developing children (who were reported by parents to be righthanded individuals) demonstrated the following scores: Overall
$\mathrm{MHI}=-0.036$, SDB $\mathrm{MHI}=-0.475$, Object $\mathrm{MHI}=0.403$. A Fisher's exact test of frequencies revealed a significant interaction of handedness and target type $(p<.0001)$.

\subsubsection{Children with autism spectrum disorder}

Mean Handedness Index scores (MHI) were calculated for target categories and overall strength of handedness. Typically developing children who were reported by parents and teachers to be righthanded individuals demonstrated the following scores: Overall $\mathrm{MHI}=0.051, \mathrm{SDB} \mathrm{MHI}=0.015$, Object $\mathrm{MHI}=0.051$. A Fisher's exact test revealed no significant interaction of handedness and target type $(p=1.0)$.

\subsection{Total frequencies of hand actions}

For TD children, SDBs accounted for $29.1 \%$ of unimanual actions, while object actions accounted for $70.9 \%$ of total unimanual actions. Similarly, for children with ASD, SDB targets accounted for $22.8 \%$ of unimanual actions, while object targets accounted for $77.2 \%$ of unimanual actions. These percentage of actions for objects and the self were not significantly different between the two experimental groups, $x^{2}(1)=0.65, p=.42$.

\section{Discussion}

\subsection{Unimanual actions to objects}

Based on the findings from the present study, right-handed TD boys and right-handed boys with ASD expressed different patterns of actions to objects. All TD boys demonstrated a significant right-handed dominance for actions to objects at both the individual level, replicating previously reported findings in both great apes [78,79] and children [56]. One interpretation of this seemingly robust pattern is that it represents an early evolutionary neural division of labor, such that the left hemisphere and right hand are preferentially engaged for hand actions for skilled sequences of hand actions (e.g. tool use) and language processes. A left hemisphere dominance for action sequences that underlie both tool use and language processes may be related to why stronger hand dominance has often been reported to correlate with earlier language acquisition [31] and the successful hemispheric specialization for language [1]. However, regardless of any causal relationship underlying hand preference, the results suggest that for typically developing boys, hand preference is influenced by the target to which the manual action is directed.

In contrast to the TD boys, only two of the four boys with ASD demonstrated a significant hand dominance for actions to objects and the direction of bias was split. While one boy with ASD expressed a relatively strong right-handed bias for actions to objects $(\mathrm{HI}=.41)$, the other expressed a relatively weak left-handed 


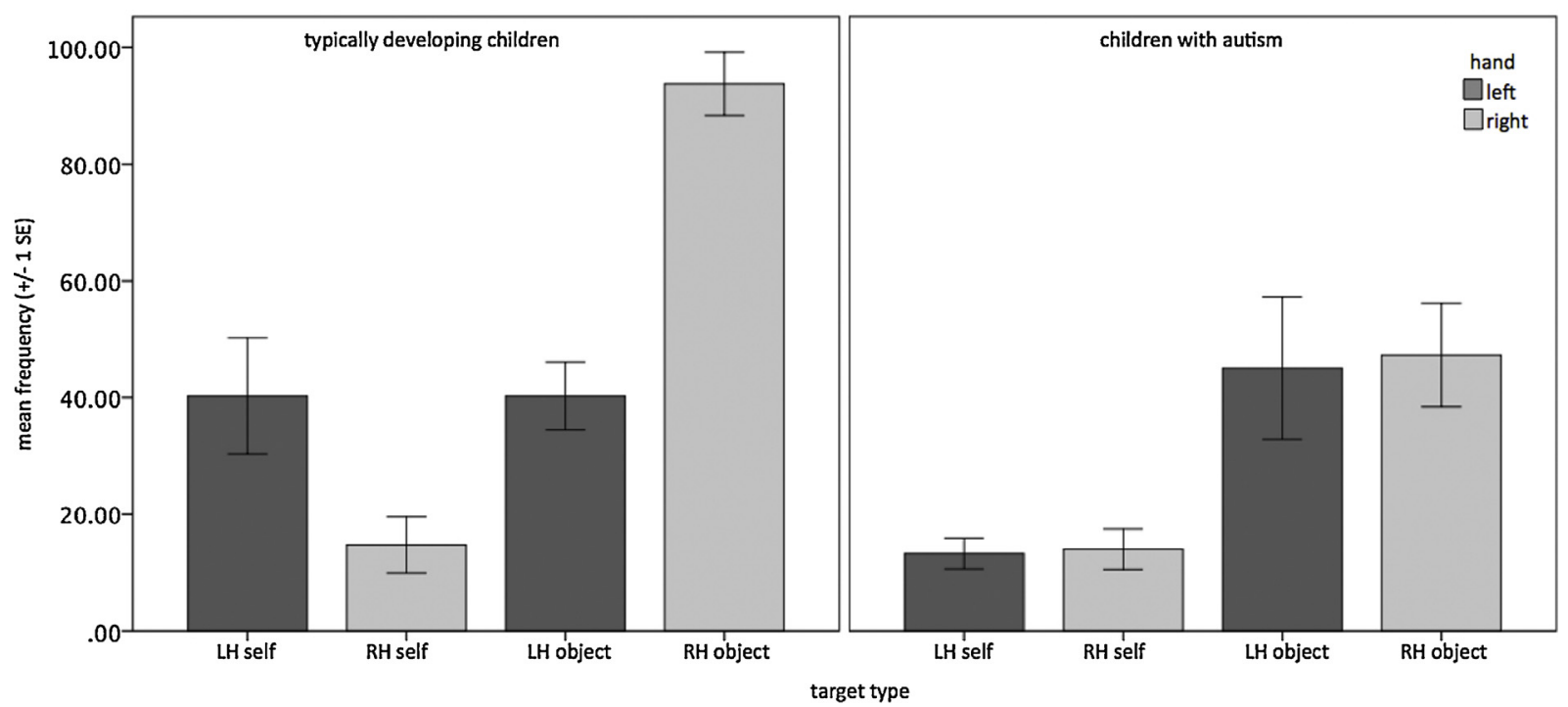

Fig. 1. Group results for the interaction of hand and action type (self, object). Mean frequencies for typically developing child results are displayed in the left panel and results from children with autism are displayed in the right panel.

preference $(\mathrm{HI}=-.24)$. The two remaining boys with ASD showed no lateral preference for actions for objects. This result is consistent with arguments that ASD is characterized by reduced cerebral lateralization [e.g. 84,85] and marked by reduced strength of handedness for object manipulation [e.g. 33-37].

\subsection{Unimanual actions to self}

Few studies have investigated hand behaviors outside of object manipulation. The studies that have considered hand actions for different target types have revealed mixed-handedness for unimanual actions to animate targets that pooled actions to the self and to social partners $[55,56,78,79]$. However, the present study addressed SDBs in isolation, considering the possibility that hand actions to social partners and hand actions to the self, may be driven by different processes. In humans, SDBs are often used as an index of emotional arousal with regards to the stress response and have been considered the manifestation of 'emotional leakage' [86] and as a result may have invoked more right hemisphere processing compared with actions associated with objects. Analyses revealed that three of the four TD boys demonstrated a significant left hand bias for SDBs, while one TD boy expressed no significant lateral preference, although a non-significant right hand preference was recorded. One interpretation of this pattern of results is that SDBs are preferentially controlled by the right hemisphere in typically developing children. These findings are consistent with studies that have reported a left-handed preference for self-directed face touching in adults who were otherwise right-handed [87], and a further study that revealed that individuals reflexively raise their non-dominant hand to protect their faces [88]. Although untested in the current study, it is possible that SDBs might represent displacement behaviors found to be correlated with levels of stress in studies of both human and nonhuman primates [89].

Although there is a paucity of human studies relating to this subject, SDBs have been associated with frustration, uncertainty and anxiety in social conflict situations in a variety of primate species [for a review see 90]. One study of chimpanzee SDBs demonstrated a significant group-level increase in self-scratching with increased task complexity [91]. Interestingly, lateral patterns associated with SDBs have also been identified in non-human primates. For example, rehabilitated orangutans exhibited a significant group-level lateralized preference for left-handed scratching and for the fine manipulation of parts of the face [92]. A further study of chimpanzees reported that while self-directed scratching showed no hand preference, there was a significant bias for scratching on the left side of the body. The authors postulated that this behavior was the manifestation of a right hemisphere dominant role in the regulation of the autonomic nervous system during arousal [93, but see 94 for complementary methodological approaches].

The left hand bias for SDBs in TD children who are otherwise right-handed children, could be acting as a biomarker for heightened emotional processing. Tasks undertaken as part of the ADOS involved active role-play with the clinician and timed problem solving. Increased levels of task complexity or novel challenges (e.g. improvisation) may have increased stress levels, resulting in an increase of right hemisphere emotional processing, eliciting left-handed actions directed to the self. This interpretation is consistent with the prevailing theories of social-emotional processing in humans. The right hemisphere hypothesis considers the right hemisphere to be dominant in all forms of emotional expression and perception [e.g. 56]. Additionally, the valence theory [e.g. 57, $58]$ has garnered support in a number of noninvasive behavioral studies including dichotic listening tasks using affective stimuli $[95,96]$ and in divided visual field studies using facial emotion stimuli [97-99], revealing a left hemisphere dominance for positive affect and a right hemisphere dominance for negative affect [e.g. $100,101]$.

The results of the boys with ASD were markedly different from those of the TD boys. While three of the four TD boys revealed handedness index (HI) scores for SDBs indicating strong left hand biases (ranging between -0.42 and -0.72 ). All four boys with ASD revealed no hand preference for SDBs with almost equal proportions of left and right hand actions directed to the self and $\mathrm{HI}$ scores ranging from -0.09 to +0.07 . Common symptoms of ASD include impairments with language and social processes yet to date, research of cerebral asymmetries for function tends to be been devoted almost exclusively to understanding structural asymmetries in language association areas [for a review see 35]. The influence of cerebral dominance for emotive processing associated with hand dominance has not been thoroughly investigated. These findings suggest that handedness for SDBs may engage the opposite hemisphere to that controlling object manipulation and language processing and as such, can provide an additional and complementary marker of cognitive function and a further indirect measure of strength of cerebral lateralization. 


\subsection{Target-dependent unimanual handedness}

Although the present study could not assess whether left hand biased SDBs and right hand biased actions for objects in typically developing boys were a direct manifestation of right and left hemisphere processing respectively, it is an important consideration for future functional imaging studies. A functional dissociation between hand preference for controlling hand actions for object manipulation and SDBs is consistent with an evolutionary functional distinction between the two hemispheres such that the left hemisphere is dominant for structured sequences of actions (e.g. tool use and language), and the right hemisphere is dominant for actions that are the manifestation of emotive processing (fight or flight) [2]. As such, handedness strength measures across functionally different targets may be a valuable behavioral marker of successful hemispheric functional lateralization across both hemispheres. Additionally, a lack of hand preference for functionally distinct targets may serve as a useful biomarker for atypical lateralization of cerebral function and thus decreased cognitive function.

\section{Conclusions}

The systematic observation of spontaneous naturalistic behavior across functionally disparate target-types remains a largely un-tapped area of investigation. To date, studies of handedness have been confounded by disparate investigative approaches across fields of study hindering comparative studies and reproducibility of findings. While we highlight a lack of consistency in approaches to studies of handedness, we purport that naturalistic hand actions provide a rich, observable behavior that may be a valuable marker of decreased cognitive function. Observational approaches of naturalistic hand behavior allow for flexible data capture across different contexts allowing for the study of an ethologically valid set of hand activities. Additionally, studies of naturalistic behavior afford greater flexibility for collecting data from participants of all ages, allowing for the early detection of children's weak hand dominance patterns.

In the future, functional imaging of the neural processing underpinning hand actions in humans and a systematic inventory of typical behavioral patterns could be used to elucidate the trajectory of typical hand strength development from birth to adulthood. Additionally, the investigation of lateralized motor behaviors of children with different cognitive disorders and delays may help to identify early disruptions to the typical development of cerebral lateralization of basic sensory motor processes that have cascading consequences for the development of higher cognitive functions. Because early motor deficits are not specific to autism, a systematic analysis of behavioral observations of typically developing children and children with developmental delays and/or disorders is necessary to understand the interaction between neurodevelopment, behavior and prognosis. This study introduces one quantitative, objective approach to the investigation of handedness that can be employed to evaluate handedness across different human and non-human primate populations, offering an opportunity to further both developmental and evolutionary aspects of human handedness.

\section{Acknowledgements}

We are grateful to the parents, teachers and children of Livingstone Primary School for their participation and to Dr. Kristelle Hudry for conducting diagnostic testing. We gratefully acknowledge funding support provided in part by European Grants: FP6-NEST-029088(ANALOGY), FP7-MC-ITN-289404(ACT),
ESRC grant RES-062-23-2721 and the University of Westminster's Psychology Publication Stimulus Scheme.

\section{References}

[1] Toga AW, Thompson PM. Mapping brain asymmetry. Nat Rev Neurosci $2003 ; 4: 37-48$

[2] MacNeilage PF, Rogers LJ, Vallortigara G. Origins of the left and right brain. Sci Am 2009;301:60-7.

[3] Rogers LJ, Andrew RJ. Comparative vertebrate lateralization. Cambridge: Cambridge University Press; 2002.

[4] Frasnelli E, Vallortigara G, Rogers LJ. Left-right asymmetries of behaviour and nervous system in invertebrates. Neurosci Biobehav Rev 2012;36:1273-91.

[5] Rogers LJ, Zucca P, Vallortigara G. Advantages of having a lateralized brain. Proc R Soc B 2004;271(6):S420-2.

[6] Tommasi L. Mechanisms and functions of brain and behavioural asymmetries. Philos Trans R Soc B 2009;364:855-9.

[7] Vallortigara G, Rogers LJ. Survival with an asymmetrical brain: advantages and disadvantages of cerebral lateralization. Behav Brain Sci 2005;28:575-633.

[8] Santrock JW. Motor, sensory, and perceptual development. In: Ryan M, editor. A topical approach to life-span development. Boston: McGraw-Hill Higher Education; 2008. p. 172-205.

[9] Hellige JB. Unity of thought and action: varieties of interaction between the left and right hemispheres. Curr Dir Psychol Sci 1993;2:21-5.

[10] Broca P. Sur le siège de la faculté du langage articulé. Bull Soc Anthropol Paris 1865;6:377-93

[11] Wernicke C. Der aphasiche Symptomenkomplex: eine psychologische Studie auf anatomischer Basis. Breslau: Cohen and Weigert; 1874.

[12] McManus IC. Right hand, left hand: the origins of asymmetry in brains, bodies, atoms, and cultures. London: Weidenfeld and Nicolson; 2002.

[13] Foundas AL, Leonard CM, Heilman KM. Morphologic cerebral asymmetries and handedness: the pars triangularis and planum temporal. Arch Neurol 1995;52(5):501-8.

[14] Knecht S, Dräger B, Deppe M, Bobe L, Lohmann H, Flöel A, et al. Handedness and hemispheric language dominance in healthy humans. Brain 2000; $123: 2512-8$

[15] Meador KJ, Loring DW, Lee K, Hughes M, Lee G, Nichols M, et al. Cerebral lateralization: relationship of language and ideomotor praxis. Neurology 1999;53(9):2028-31.

[16] Cochet H, Jover M, Vauclair J. Hand preference for pointing gestures and bimanual manipulation around the vocabulary spurt period. J Exp Child Psychol 2011;110(3):393-407.

[17] Esseily R, Jacquet AY, Fagard J. Handedness for grasping objects and pointing and the development of language in 14-month-old infants. Laterality 2011;16(5):565-85

[18] Szaflarski JP, Binder JR, Possing ET, McKiernan KA, Ward BD, Hammeke TA. Language lateralization in left-handed and ambidextrous people: fMRI data. Neurology 2002;59:238-44.

[19] Hopkins WD. On the other hand: Statistical issues in the assessment and interpretation of hand preference data in nonhuman primates. Int J Primatol 1999;20:851-66.

[20] Oldfield RC. The assessment and analysis of handedness: the Edinburgh inventory. Neuropsychologia 1971;9:97-113.

[21] Healy JM, Liederman J, Geschwind N. Handedness is not a unidimensional trait. Cortex 1986;22:33.

[22] Beaton AA. The determinants of handedness. In: Hugdahl K, Davidson RJ, editors. Brain asymmetry. 2nd ed. MIT Press; 2003. p. p105-58.

[23] Annett M. The growth of manual preference and speed. Br J Psychol 1970;61(4):545-58.

[24] Gudmundsson E. Lateral preference of preschool of primary school children. Percept Mot Skills 1993;77:819-28.

[25] Ingram D. Motor asymmetries in young children. Neuropsychologia 1975;13(1):95-102.

[26] Hervé PY, Crivello F, Perchey G, Mazoyer B, Tzourio-Mazoyer. Handedness and cerebral anatomical asymmetries in young adult males. Neuroimage 2006;29:1066-79.

[27] Dane S, Balci N. Handedness, eyedness and nasal cycle in children with autism. Int J Dev Neurosci 2007;25:223-6.

[28] Lewin J, Kohen D, Mathew G. Handedness in mental handicap: investigation into populations of Down's syndrome, epilepsy and autism. Br J Psychiatry 1993;163:674-6.

[29] Satz P, Soper HV, Orsini DL, Henry RR, Zvi JC. Handedness subtypes in autism. Psychiatr Ann 1985;15:447-50.

[30] Soper HV, Satz P, Orsini DJ, Henry RR, Zvi JC, Schulman M. Handedness patterns in autism suggest subtypes. J Autism Dev Disord 1986;16(2):155-67.

[31] Leask SJ, Crow TJ. Word acquisition reflects lateralization of hand skill. Trends Cogn Sci 2001;5(12):513-6.

[32] Kastner-Koller U, Deimann P, Bruckner J. Assessing handedness in preschoolers: construction and initial validation of a hand preference test for 4-6-year-olds. Psychol Sci 2007;49(3):239-54.

[33] Crow TJ, Crow LR, Done DJ, Leask S. Relative hand skill predicts academic ability: global deficits at the point of hemispheric indecision. Neuropsychology 1998;36(12):1275-82

[34] Delcato CH. Neurological organization and reading. Illinois: Springfield; 1966. 
[35] Orton ST. Reading, writing, and speech problems in children. New York: Norton; 1937.

[36] Yeo RA, Gangestad SW, Thoma RJ. Developmental instability and individual variation in brain development: implications for the origin of neurodevelopmental disorders. Curr Dir Psychol Sci 2007;16(5):245-9.

[37] Yeo RA, Gangestad SW, Thoma R, Shaw P, Repa K. Developmental instability and cerebral lateralization. Neuropsychology 1997;11(4):552-61.

[38] World Health Organisation. 2006 WHO Multicentre Growth Reference Study Group WHO Motor Development Study: Windows of achievement for six gross motor development milestones. Acta Paediatr Suppl 1992;450:86-95.

[39] Tager-Flusberg H, Caronna E. Language disorders: autism and other pervasive developmental disorders. Pediatr Clin North Am 2007;54(3):469-81.

[40] Wetherby A, Woods J, Allen L, Cleary J, Dickson H, Lord C. Early indicators of autism spectrum disorders in the second year of life. J Autism Dev Disord 2004;34(5):473-93.

[41] Mandell DS, Novak MM, Zubritsky CD. Factors associated with age of diagnosis among children with autism spectrum disorders. Pediatrics 2005; $116: 1480-6$

[42] Satz P, Nelson L, Green M, Ambiguous-handedness: incidence in a non-clinical sample. Neuropsychologia 1989;27(10):1309-10.

[43] Lindell AK, Hudry K. Atypicalities in cortical structure, handedness, and functional lateralization for language in autism spectrum disorders. Neuropsychol Rev 2013;23:257-70.

[44] Rodriguez A, Kaakinen M, Moilanen I, Taanila A, McGough JJ, Loo S, et al. Mixed-handedness is linked to mental health problems in children and adolescents. Pediatrics 2010;125(2):340-8.

[45] Knaus TA, Silver AM, Kennedy M, Lindgren KA, Dominick KC, Siegel J, et al. Language laterality in autism spectrum disorder and typical controls: a functional volumetric, and diffusion tensor MRI study. Brain Lang 2010;112(2):113-20.

[46] Elsabbagh M, Johnson MH. Getting answers from babies about autism. Trends Cogn Sci 2010;14:81-7.

[47] Thomas MSC, Knowland VCP, Karmiloff-Smith A. Mechanisms of developmental regression in autism and the broader phenotype: a neural network modeling approach. Psychol Rev 2011;118(4):637-54.

[48] Leonard HC, Elsabbagh M, Hill EL, the BASIS team. Early and persistent motor difficulties in infants at-risk of developing autism spectrum disorder: a prospective study. Eur J Dev Psychol 2014;11(1):18-35.

[49] Fagard J, Marks A. Unimanual and bimanual tasks and the assessment of handedness in toddlers. Dev Sci 2000;3(2):137-47.

[50] Hinojosa T, Sheu CF, Michel GF. Infant hand-use preferences for grasping objects contributes to the development of a hand-use preference for manipulating objects. Dev Psychobiol 2003;43(4):328-34

[51] Potier C, Meguerditchian A, Fagard J. Handedness for bimanual coordinated actions in infants as a function of grip morphology. Laterality 2013;18(5):576-93.

[52] Cochet H, Vauclair J. Pointing gestures produced by toddlers from 15 to 30 months: different functions, hand shapes and laterality patterns. Infant Behav Dev 2010;33:432-42.

[53] Cochet $\mathrm{H}$, Vauclair J. Pointing gesture in young children: hand preference and language development. Gesture 2010;10(2/3):129-49.

[54] Jacquet AY, Esseily R, Rider D, Fagard J. Handedness for grasping objects and declarative pointing: a longitudinal study. Dev Psychol 2011;54(1):36-46.

[55] Marchant LF, McGrew WC, Eibl-Eibesfeldt I. Is human handedness universal? Ethological analyses from three traditional cultures. Ethology 1995;101:239-58.

[56] Forrester GS, Quaresmini C, Leavens DA, Mareschal D, Thomas MSC. Human handedness: an inherited evolutionary trait. Behav Brain Res 2013;237:200-6.

[57] Borod JC, Caron HS, Koff E. Left-handers and right-handers compared on performance and preference measures of lateral dominance. Br J Psychol $1984 ; 75: 177-86$.

[58] Davidson RJ, Ekman P, Saron CD, Senulis JA, Friesen WV. Approachwithdrawal and cerebral asymmetry: emotional expression and brain physiology I. J Pers Soc Psychol 1990;58:330-41.

[59] Davidson RJ. Cerebral asymmetry, emotion, and affective style. In: Davidson RJ, Hugdahl K, editors. Brain asymmetry. Cambridge, MA: MIT Press; 1995. p. 361-89.

[60] Ekman P, Friesen WV. Nonverbal leakage and clues to deception. Psychiatry 1969;32:88-105

[61] Ekman P. Biological and cultural contributions to body and facial movements. In: Blacking J, editor. A.S.A. monograph 15 the anthropology of the body. London: Academic Press; 1977.

[62] Ekman P, Freisen WV. Hand movements. J Commun 1972:353-74

[63] Chrousos GP, Gold PW. The concepts of stress and stress system disorders: overview of physical and behavioral homeostasis. J Am Med Assoc 1992;267:1244-52.

[64] MacWhinney B. The TalkBank project. In: Beal J, Corrigan K, Moisl L, editors. Creating and digitizing language corpora: synchronic databases, Houndsmill, Basingstoke, vol. 1. Hampshire: Palgrave-Macmillan; 2007.

[65] Forrester GS. A multidimensional approach to investigations of behavior: revealing structure in animal communication signals. Anim Behav 2008;76:1749-60

[66] Bates E, O'Connell B, Vaid J, Sledge P, Oakes L. Language and hand preference in early development. Dev Neuropsychol 1986;2:1-15.

[67] McManus IC, Sik G, Cole DR, Mellon AF, Wong J, Kloss J. The development of handedness in children. Br J Dev Psychol 1988;6(3):257-73.
[68] Whiteley P, Todd L, Carr K, Shattock P. Gender ratios in autism, asperger syndrome and autism spectrum disorder. Autism Insights 2010;2: $17-24$.

[69] Hutchins E. Cognition in the wild. Cambridge, MA: MIT Press; 1995.

[70] Hutchins E. The distributed cognition perspective on human interaction. In: Enfield NJ, Levinson SC, editors. Roots of human sociality: culture, cognition and interaction. Oxford: Berg Publishers; 2006. p. 375-98.

[71] Johnson CM. Distributed primate cognition: a review. Anim Cogn 2001;3(4):167-83.

[72] Altmann J. Observational study of behaviour sampling methods. Behaviour 1974;49:227-67.

[73] Lord C, Risi S, Lambrecht L, Cook Jr EH, Leventhal BL, DiLavore PC, et al. The autism diagnostic observation schedule-generic: a standard measure of social and communication deficits associated with the spectrum of autism. J Autism Dev Disord 2000;30(3):205-23.

[74] Fagard J, Lockman JJ. The effect of task constraints on infants' (bi)manual strategy for grasping and exploring objects. Infant Behav Dev 2005;28(3):305-15.

[75] Flowers K. Handedness and controlled movement. $\mathrm{Br} J$ Psychol 1975;66:49-52.

[76] Provins KA, Glencross DJ. Handwriting, typewriting and handedness. QJ Exp Psychol 1968;10:282-9.

[77] Fagot J, Vauclair J. Manual laterality in nonhuman primates: a distinction between handedness and manual specialization. Psychol Bull 1991:109:76-89.

[78] Forrester GS, Leavens DA, Quaresmini C, Vallortigara G. Target animacy influences gorilla handedness. Anim Cogn 2011;14:903-7.

[79] Forrester GS, Quaresmini C, Leavens DA, Spiezio C, Vallortigara G. Target animacy influences chimpanzee handedness. Anim Cogn 2012;15:1121-7.

[80] Hopkins WD, Fernandez-Carriba S, Wesley MJ, Hostetter A, Pilcher D, Poss S. The use of bouts and frequencies in the evaluation of hand preferences for a coordinated bimanual task in chimpanzees (Pan troglodytes): an empirical study comparing two different indices of laterality. J Comp Psychol 2001;115:294-9.

[81] McGrew WC, Marchant LF. On the other hand: current issues in and metaanalysis of the behavioral laterality of hand function in nonhuman primates. Yearbook Phys Anthropol 1997;40:201-32.

[82] Hurlbert SH. Pseudoreplication and the design of ecological field experiments. Ecol Monogr 1984:54:187-211.

[83] Palmer AR. Reply to Hopkins and Cantalupo: chimpanzee right-handedness reconsidered-sampling issues and data presentation. Am J Phys Anthropol 2003; $121: 382-4$

[84] Hauck JA, Dewey D. Hand preference and motor functioning in children with autism. J Autism Dev Disord 2001;31(3):265-77.

[85] Tsai LY. Brief report: the development of hand laterality in infantile autism. J Autism Dev Disord 1984;14(4):447-50.

[86] Waxer PH. Nonverbal cues for anxiety: an examination of emotional leakage. J Abnorm Psychol 1977:86(3):306-14

[87] Dimond S, Harries R. Face touching in monkeys, apes and man: evolutionary origins and cerebral asymmetry. Neuropsychologia 1984;22:227-33.

[88] Coren S. The left-hander syndrome: the causes and consequences of lefthandedness. New York: Random House; 1992.

[89] Triosi A. Displacement activities as a behavioral measure of stress in nonhuman primates and human subjects. Stress 2002;5(1):47-54

[90] Maestripieri D, Schino G, Aureli F, Triosi A. A modest proposal: displacement activities as an indicator of emotions in primates. Anim Behav 1992;44(5):967-79.

[91] Leavens DA, Aureli F, Hopkins WD, Hyatt CW. The effects of cognitive challenge on self-directed behaviors by chimpanzees (Pan troglodytes). Am J Primatol 2001;55:1-14.

[92] Rogers LJ, Kaplan G. Hand preferences and other lateral biases in rehabilitated orangutans. Pongo pygmaeus pygmaeus. Anim Behav 1995;51:13-25.

[93] Hopkins WD. Lateralized Scratching in Chimpanzees (Pan troglodytes): evidence of a functional asymmetry during arousal. Emotion 2006;6(4): 553-9.

[94] de Souza Silva MA, Topic B, Lamounier-Zepter V, Huston JP, Tomaz C, Barros M. Evidence for hemispheric specialization in the marmoset (Callithrix penicillata) based on lateralization of behavioral/neurochemical correlations. Brain Res Bull 2005;74:416-28.

[95] Bryden MP, Free T, Gagne S, Groff P. Handedness effects in the detection of dichotically-presented words and emotions. Cortex 1991;27(2): 229-35.

[96] McFarland RA, Kennison R. Handedness affects emotional valence asymmetry. Percept Mot Skills 1989;68(2):435-41

[97] Everhart DE, Harrison DW, Crews WD. Hemispheric asymmetry as a function of handedness-perception of facial affect stimuli. Percept Mot Skills 1996;82(1):264-6.

[98] Natale M, Gur RE, Gur RC. Hemispheric asymmetries in processing emotional expressions. Neuropsychologia 1983;21(5):555-65.

[99] Önal-Hartmann C, Pauli P, Ocklenburg S, Güntürkün O. The motor side of emotions: investigating the relationship between hemispheres, motor reactions and emotional stimuli. Psychol Res 2012;76(3):311-6.

[100] Elias LJ, Bryden MP, Bulman-Fleming MB. Footedness is a better predictor than is handedness of emotional lateralization. Neuropsychologia 1998;36(1):37-43.

[101] Shamay-Tsoory SG, Lavidor M, Aharon-Peretz J. Social learning modulates the lateralization of emotional valence. Brain Cogn 2008;67(3):280-91. 